\title{
Social network positions, trust behavior and its neural mechanisms in young adolescents
}

H. Sijtsma ${ }^{1}$, M. van Buuren ${ }^{1}$, M. Hollarek ${ }^{1}$, R. J. Walsh ${ }^{1}$, N.C. Lee ${ }^{1}$, B.R. Braams ${ }^{1}$, L. Krabbendam $^{1}$

${ }^{1}$ Section of Clinical Developmental Psychology, Research Institute LEARN!, Institute of Brain and Behavior, Faculty of Behavioral and Movement Sciences, Vrije Universiteit Amsterdam

Corresponding author:

Hester Sijtsma

h.sijtsma@vu.nl

Word count: 4992 words 


\section{Abstract}

Assessing the trustworthiness of others to learn who you can and cannot trust is important for social relationships. During early adolescence, the social environment becomes increasingly complex. The number and nature of one's friendships may help to shape, and may be facilitated by, adequate trust behavior. In the current fMRI study, we investigated in 49 young adolescents (Mage=12.8, SDage=0.4, 18 boys) the ability to adapt trust behavior when interacting with an untrustworthy partner as well as the neural mechanisms of trust using a trust game. Furthermore, we examined how trust behavior, the neural mechanisms of trust, and the position in a social network are related. Results indicate that adolescents decreased their trust behavior throughout the game. fMRI analysis showed no increased activity in regions of interest when making trust decisions. When receiving feedback, increased activity in the cognitive control and reward network was shown. Furthermore, less central social network positions (eigencentrality) were associated with lower overall trust behavior, which was the better strategy in the current interaction. In contrast, eigencentrality was not related to the adaptation of trust behavior or to brain activity. The current study provides insights in trust behavior and the neural mechanisms of trust in young adolescents.

Key words: Adolescence, trust behavior, fMRI, friendships, social network analysis 


\section{Introduction}

During social interactions, it is essential to assess the trustworthiness of the interaction partner and to adapt one's own trust behavior accordingly. Early adolescence is an important period to learn who can and cannot be trusted, because the social context becomes more complex, and relationships with individuals outside the family become increasingly important (Brown \& Larson, 2009; Steinberg \& Morris, 2001). Research in adolescents shows age-related increases in trusting others (Fett et al., 2012; Sutter \& Kocher, 2007; van den Bos et al., 2010). Furthermore, young adults are able to adapt their trust behavior when the partner's trust behavior differs from what was expected (Delgado et al., 2005), and this flexibility improves during adolescence (Lee et al., 2016). In this study we investigated the ability to adapt one's trust behavior towards an untrustworthy interaction partner and the neural mechanisms of trust in young adolescents. Based on the notion that one's friendships may help to further shape adequate trust behavior, we also examined the relationship between the ability to adapt trust behavior, the neural mechanisms of trust and real-life friendships.

Trust behavior and underlying neural mechanisms can be investigated by using a trust game (Berg et al., 1995; King-Casas et al., 2005). In this paradigm, the participant (the trustor) shares money with the interaction partner (the trustee). The amount invested is tripled and received by the partner who then decides how much to share with the trustor. The game can be repeated for multiple rounds. The change in investments in response to the partner's behavior over the course of the game indicates the ability to adapt trust behavior while the average investment indicates overall trust. In the trust game mentalizing processes are involved as the mental states and intentions of the partner are assessed (Fett et al., 2012). Also, reward and conflict monitoring processes are addressed when one's trust behavior is reciprocated and when the partner's behavior 
is different than anticipated (King-Casas et al., 2005). Neuroimaging studies using the trust game have shown activity within areas related to mentalizing such as the temporal-parietal junction (TPJ), posterior cingulate cortex, precuneus and the medial prefrontal cortex (mPFC) (Delgado et al., 2005; Fett et al., 2012; Van Den Bos et al., 2009; van den Bos, van Dijk, Westenberg, et al., 2011). Furthermore, brain areas associated with reward processing such as the orbitofrontal cortex, the caudate and the insula, as well as regions involved in conflict monitoring, including the dorsolateral prefrontal cortex (dIPFC) and the anterior cingulate cortex (ACC), are activated during the trust game (Delgado et al., 2005; Fett et al., 2012; Lemmers-Jansen et al., 2017; Van Den Bos et al., 2009).

One method to examine the ability to adapt trust behavior is to manipulate the behavior of the hypothetical partner as untrustworthy using an algorithm. The partner can behave in such a manner that the higher the trustor's investment, the less the partner returns. To avoid losing money, the trustor ought to reduce their investment. Research showed a greater decrease of trust behavior with age in a sample between 13 and 49 years old (Fett et al., 2012). The decrease in trust behavior was accompanied by increased ACC activity, possibly indicating conflict monitoring between the desire to show trust behavior and the partner's actual untrustworthy behavior (Fett et al., 2012). In another study, adolescents with high perspective-taking abilities showed a greater adaptive decrease in investments compared to adolescents with low perspective-taking abilities (age 13-18: Fett et al., 2014). However, the response to an untrustworthy partner and the neural mechanisms of trust in young adolescents are as yet unexplored.

The number and nature of social relationships in one's social network and trust behavior may affect each other. That is, trust behavior may facilitate successful engagement in social interactions and relationships, and in turn, social interactions and social relationships may shape 
the development of adequate trust behavior. For example, in previous studies in companies, it was shown that trustworthy behavior is related to cohesiveness in social networks: people in close, cohesive networks are more inclined to show trustworthy behavior, partly because untrustworthy behavior is noticed sooner in a close network (Burt et al., 2013). Also, in another study, a positive association between trust behavior and social cohesion in a classroom was found (van den Bos et al., 2018). Furthermore, the number of social relationships has been positively associated to overall trust (Buskens, 1998), although in another study, this association was not observed (van den Bos et al., 2018).

So far, little is known about the relationship between social networks positions and the ability to adapt trust behavior. In a previous study, we found that late adolescents with less central social network positions were better able to adaptively increase their trust behavior than more central adolescents (Sijtsma et al., 2020). While speculative, we suggested that adolescents with less central positions are more motivated to understand the behavior and intentions of others, because their position is less powerful. This is based on the power-dependence relation theory that explains that people lower in power depend on more influential and central people to achieve goals, while more central people are less dependent on and less attentive to others (Emerson, 1962). Related to this, research has shown that power and the ability to influence others is negatively related to perspective taking (Galinsky et al., 2006; Galinsky et al., 2016). Furthermore, several studies have investigated how the relation between measures of one's social network and social behavior is instantiated in the brain (Bickart et al., 2012; Dunbar, 1998; Falk \& Bassett, 2017; Powell et al., 2012). For example, in comparison to adolescents with dense friendship networks, adolescents with less dense friendship networks showed stronger connectivity between areas related to mentalizing during a social exclusion task when they were excluded compared to when 
they were not excluded, possibly indicating the use of more mentalizing resources during social exclusion (Schmälzle et al., 2017). Together, these studies point to the intricate relationships between social networks, social behavior and the brain areas involved in social behavior.

In the current study, we examined the relationship between real-life social network measures, trust behavior and neural mechanisms of trust in young adolescents. The first aim was to investigate whether they are able to adapt trust behavior when playing with an untrustworthy partner and to investigate the neural mechanisms of trust. As children are able to differentiate between trustworthy, neutral and untrustworthy partners (Van den Bos, van Dijk, \& Crone, 2011), it is hypothesized that young adolescents will decrease their trust behavior over the course of the game. Additionally, during trust decisions increased activity (relative to the control condition) is expected in areas involved in mentalizing, while during the processing of the returned investment, increased activity is expected in areas related to control and reward processes. The second aim was to examine the relationship between adolescents' position in their friendship network, trust behavior and associated brain activity. Based on the power-dependence relation theory and our previous findings, we expect adolescents with more peripheral social positions to show a greater decrease in trust behavior over time and less overall trust behavior. Also, during trust decisions, we expect more peripheral network positions to be positively associated with activity in areas involved in mentalizing.

\section{Method}

\section{Participants}

The current study was part of the project \#SOCONNeCT. Six-hundred ninety-two participants were included in the \#SOCONNeCT study and six waves of data collection were 
carried out in school classes. They were invited to additional studies of the \#SOCONNeCT project, including the current fMRI study. Three-hundred seventy adolescents showed interest of which $44 \%$ were excluded due to orthodontic braces, $37 \%$ refrained from participation after all and $19 \%$ failed to reply. Eighty-six participants were included in the current study. Two participants refrained from participation because they were too anxious at the start of the scan session. Fifteen participants were excluded due to too much movement (>3 mm). To secure reliable social network measures, the participation rate within a class must be above 75\% (Cillessen \& Marks, 2011; Marks et al., 2013). Twenty participants were excluded because they were in classes with a participation rate below the cut-off of $75 \%$. Details on the participation rate per class can be found in the supplementary materials. Forty-nine participants were included in the final analyses (Mage $=12.8$, SDage $=0.4,18$ boys $)$. Participants received monetary compensation for participation and a monetary bonus based on their winnings during the trust game. The current study was approved by the Scientific and Ethical Review Board of the Faculty of Behavioral and Movement Sciences of the Vrije Universiteit Amsterdam.

\section{Procedure}

Participants and their parent(s)/caregiver(s) came to the MRI scanner location and both gave written informed consent. To get acquainted with the response buttons the participants played a practice version of the trust game in the mock scanner. In this practice version, participants played against themselves and their return was equal to their investment to prevent learning effects. Next, the participant laid down in the MRI scanner and was instructed to play a trust game with a computer animation. The participants could view the trust game projected on a screen through a mirror on the head coil. Button boxes were used to perform the trust game. The MRI procedure 
started with a resting state period, followed by a task, a structural scan and ended with the trust game. The total test session lasted 45 minutes.

\section{Measures}

Trust game and trust game questionnaire

The trust game consisted of 27 experimental trials and 27 control trials. An experimental trial (see Figure 1) started with a cue (1 second) that indicated an investment between zero and ten euros could be made. Next, the participant made an investment ( 3 seconds) after which the investment was shown (1 second). The numbers on the screen turned green after the investment was made. The right index and middle finger were used to move the cursor left and right and the left index finger was used to select an amount. The invested amount was tripled before it was received by the partner. After a waiting period (1 to 5 seconds, mean jitter 3 seconds) the participant saw a fixation cross for 1.5 to 5.5 seconds (mean jitter 3.5 seconds). Next, a screen was displayed for 3 seconds showing the amount of money the partner kept for himself and the amount the participant received from the partner, followed by the total result for that specific trial $(2.5$ seconds). An intertrial interval of 1.5 to 5.5 seconds was inserted before the start of the next trial, during which a fixation cross was presented. During control trials (see Figure 1) the participant selected a number instead of making an investment. The screens of a control and an experimental trial consisted of comparable visual input. After the trust game, the participant rated the partner on a scale of 0 to 10 on honesty and trustworthiness

The behavior of the partner was determined by an algorithm modeling untrustworthy behavior. The return of the partner was based on the trustor's investment multiplied by a predefined factor $(0.5,0.75$ or 1.0$)$ and rounded to the nearest 50 cents. The factor of 0.5 is the most 
untrustworthy as this would result in returning half of the trustor's investment. At the start of the game, each factor was used with equal probability, but this changed depending on the trustor's behavior. When the trustor increased the investment compared to the previous trial, the probability of a smaller return increased. The probability of factor 1.0 decreased with $5 \%$ while the factors of 0.5 and 0.75 became $2.5 \%$ more probable. In other words, the partner became more untrustworthy. After seven trials of increasing the investment, the probability of factor 0.5 was $50 \%$, the probability of factor 0.75 was $50 \%$ and the probability of factor 1 was $0 \%$ after which the factors remained stable. The factors did not change if the trustor's investment decreased or stayed the same compared to the previous trial. To test whether the behavior of the partner was mostly untrustworthy throughout the game, we calculated the number of consecutive trials in which the factor determining the return of the investment was below 1 . On average, this was the case in $72 \%$ of the consecutive trials $(15 \%-54 \%, S D=8 \%)$.

*insert Figure $1 *$

\section{$\underline{\text { Social network position }}$}

The social network position was measured by calculating the eigencentrality of each individual in the friendship network. This was done by asking the question 'Who are your friends?' to all participants of school classes participating in the \#SOCONNeCT study. A maximum of fifteen classmates could be selected. Using UCINET, the eigencentrality of each participant was calculated, which is a measure that indicates how central each person is within the network taking into account the eigencentrality of the persons to which one is connected (Borgatti et al., 2002; Robins, 2015). The eigencentrality was based on reciprocal friendships meaning that the data was 
symmetrized. This means the more central your friends are and the more reciprocal friendships you have, the higher your own eigencentrality score. The normalized eigencentrality produced by UCINET was used to control for differences in class sizes.

\section{Behavioral data analysis}

Mixed models (packages lme4 and lmerTest) in R (version 3.5.1) were used to perform behavioral data analysis (Bates et al., 2015; Kuznetsova et al., 2017). Mixed models allow the intercept and the slope to be fixed or random. A fixed intercept models the mean starting point of the investments, while a random intercept models the individual differences in starting points. Likewise, a fixed slope models the mean change of investments over time while a random slope models individual differences in the change of investments. There are repeated measurements of the same participant over time so the only level within the model is the level of the participant. First, a null model with a random intercept for the level participant was fitted. In model 1, the variable time (both the linear and the quadratic effect) and the variable gender were added as fixed effects. Gender was included as a control variable as previous research showed gender differences in trust (Van den Akker et al., 2018). Variables were kept in the model when the model fit improved. In model 2, a random slope of time was added. In model 3, the variable eigencentrality was added as z-scores in a two-way interaction with time as a fixed effect. The maximum likelihood estimation method was used to fit the models. Model comparison was done using the likelihood ratio test $(p<0.05)$ and Akaike Information Criterion (AIC) values (lower values indicate a better model fit). Bayesian Information Criterion (BIC) values for model fit are also provided for completeness. Additionally, the effect of the social network position on baseline trust was examined using a linear regression analysis. Additionally, we examined whether there are 
gender differences in baseline trust behavior using a Student's $t$-test. Baseline trust behavior is the investment made during the first trial.

\section{MRI data analysis}

Statistical Parametric Mapping 12 was used to perform MRI analyses. First, functional images were realigned to the first reference scan. Next, the structural image was co-registered to the mean functional image obtained with realignment. Then, tissue probability maps matched for the age and gender of our sample were created using the CerebroMatic toolbox (Wilke et al., 2017) and these maps were used to segment the co-registered structural image. Next, the functional and structural images were normalized to MNI space using the normalization parameters obtained with segmentation and smoothed using a $6 \mathrm{~mm}$ Gaussian kernel full width at half maximum.

The general linear model (GLM) consisted of four regressors of interest. The first regressor modeled the period in which the participant made an investment during the experimental trial (4 seconds, see Figure 1). A second regressor modeled the period in which the participant selected the number during the control trial (duration of 4 seconds). Reaction time was included as a parametric modulator to both regressors (4 seconds, see Figure 1). The regressor modeling the feedback contained the period that the participant viewed the return of the partner and viewed the totals of the specific experimental trial (duration of 5.5 seconds, see Figure 1). A fourth regressor modeled the 5.5 second period representing the feedback phase of the control trials (see Figure 1). A regressor of no interest was included consisting of the time covering the cue to invest/select and the waiting periods of the experimental trials and control trials (see Figure 1). All regressors were modeled by convolving a box-car function with a canonical hemodynamic response function (Friston et al., 1995). Six motion parameters obtained with realignment were added as regressors 
of no-interest to remove effects of motion and a high-pass filter was included to remove low frequencies not of interest (cut-off 128 seconds).

Subsequently, for each subject two contrasts images were created by contrasting the investment phase and the feedback phase of experimental trials to the equivalent phases of the control condition.

\section{Region of interest analysis}

Three brain networks were specified for a region of interest analysis using MarsBar version 0.44 toolbox (http://marsbar.sourceforge.net). These networks consisted of regions defined as 10$\mathrm{mm}$ spheres (except for the caudate for which we used a $5 \mathrm{~mm}$ sphere) centered at peak coordinates of regions associated with trust game literature (Lemmers-Jansen et al., 2017). The 'mentalizing network' was composed of the left TPJ (x, y, z = -44, -46, 29), the right TPJ (x, y, z = 45, -43, 32), and the mPFC (x, y, z = 0, 42, 6). The 'cognitive control network' entailed the dorsal ACC (x, y, $\mathrm{z}=-3,27,33)$, the right $\operatorname{dlPFC}(\mathrm{x}, \mathrm{y}, \mathrm{z}=51,18,30)$ and the left $\operatorname{dlPFC}(\mathrm{x}, \mathrm{y}, \mathrm{z}=-51,18,30)$ using mirrored coordinates of the right dlPFC. The 'reward network' entailed the left insula $(x, y, z=-$ $33,21,1)$, the right insula $(x, y, z=36,24,0)$, left caudate $(x, y, z=-7,12,-4)$ and the right caudate $(\mathrm{x}, \mathrm{y}, \mathrm{z}=6,11,5)$.

Next, for each subject and each network, mean contrast values were extracted for the investment and feedback phase. To examine the first research aim which networks were associated with trust behavior, one-sample t-tests were performed for the investment and the feedback phase. Bonferroni correction was applied separately for the investment phase and the feedback phase (alpha level becomes 0.0167). Additionally, we examined whether there were gender differences in brain activity in the three networks during the investment phase or the feedback phase using 
independent t-tests. For the second research aim, examining the relationship between adolescents' social network position with brain activity, the contrast values of the investment phase per network were regressed on the eigencentrality values. As an additional exploratory analysis, we repeated the network position analysis using contrast values of the feedback phase. Bonferroni correction was applied separately for the investment phase and the feedback phase (alpha level becomes 0.0167). Additionally, whole-brain analyses were performed to explore activity changes outside the ROIs.

\section{Results}

\section{Trust behavior}

The first research aim was to examine the ability to adapt trust behavior when interacting with an untrustworthy partner and to examine the neural mechanisms of trust in young adolescents. Descriptive results can be found in the supplementary materials. Multi-level analysis showed the best model fit for model 3 including the random slope of time and the interaction between eigencentrality and time as a fixed effect. The results of model 3 showed a negative linear effect of time on participants' trust behavior, indicating that the participants significantly decreased the investments over time. See Table 1 for the AIC and BIC values of all models and Table 2 for the description of model 3. No gender differences in baseline trust were observed ( $p=0.61$ ). The postgame questionnaire showed the average rating of the partner's honesty was 3.13 ( $s d=2.62)$ and trustworthiness was $2.93(s d=2.83)$.

The fMRI analyses showed no significant activity during the investment phase within the three networks (mean contrast value of all three networks: $p>0.0167$, see Figure 2). Second, the results showed increased activity within the cognitive control network $(t(48)=9.816, p<0.001)$ and 
the reward network $(t(48)=10.624, p<0.001)$ during the feedback phase (see Figure 2 ). No significant activity was found in the mentalizing network ( $p>0.0167$, see Figure 2$)$. Furthermore, no gender differences were found in the three networks during the investment phase or the feedback phase (mentalizing network investment phase: $p=0.81$, mentalizing network feedback phase: $p=0.93$, cognitive control network investment phase: $p=0.53$, cognitive control network feedback phase: $p=0.14$, reward network investment phase: $p=0.82$, reward network feedback phase: $p=0.54)$.

Exploratory whole-brain analyses showed increased activity during the investment phase within the bilateral occipital lobe, bilateral striatum, bilateral superior parietal lobe and the dorsal medial prefrontal cortex extending into the dorsal ACC (see Figure 3). During the feedback phase increased activity was observed within the bilateral occipital lobe, the dorsal medial prefrontal cortex extending into the dorsal ACC, dorsal lateral prefrontal cortex, bilateral striatum, bilateral insula, bilateral superior parietal lobe and, bilateral angular gyri (see Figure 4).

\footnotetext{
*insert Table $1 *$

*insert Table $2 *$

*insert Figure 2*

*insert Figure 3*

*insert Figure $4 *$
}

\section{$\underline{\text { Relationship between trust behavior and social networks }}$}

The second research aim was to examine the relationship between adolescents' position in a friendship network, trust behavior and brain activity. The results of model 3 showed a positive 
effect of eigencentrality on the overall investments, but no effect of eigencentrality on the change of investments over time (see Table 2 and Figure 5). The positive effect of eigencentrality on baseline trust just failed to reach significance $(t(47)=1.949, p=0.0572)$. Eigencentrality was not related to brain activity during either the investment phase or the feedback phase (lowest p-value was $p=0.227$.

*insert Figure 5*

\section{Discussion}

In the current study, the ability to adapt trust behavior towards an untrustworthy partner, the neural mechanisms of trust and the associations with the social network position were examined in young adolescents. Results showed that young adolescents decreased their investments over time. Brain activity analyses showed no increased activity in the mentalizing network, the cognitive control network and the reward network during the investment phase. During the feedback phase, increased activity was observed in the cognitive control network and the reward network. Furthermore, we found overall trust behavior to be positively associated with social network position, suggesting that adolescents with more peripheral positions showed less overall trust. However, social network position was not related to the adaptation of trust behavior or to brain activity.

\section{Adaptation of trust behavior and neural mechanisms of trust}

First, we examined whether young adolescents adapt their trust behavior to an untrustworthy partner and investigated the neural mechanisms of trust. In line with the hypothesis, 
young adolescents decreased their investments over time, suggesting they recognized that the partner behaved untrustworthily. Participants correspondingly gave low ratings of the partner on honesty and trustworthiness. These results are consistent with a previous study in which young adolescents showed different trust behavior towards an untrustworthy, a neutral and a trustworthy partner (Van den Bos, van Dijk, \& Crone, 2011). Based on these findings, we cautiously suggest that young adolescents understand the untrustworthy behavior and intentions of others.

Although the behavioral results suggest that young adolescents understand the intentions of others, we did not find significant neural activity changes within the mentalizing network (bilateral TPJ and mPFC) or the other two networks accompanying the investment decisions. A possible explanation for the insignificant neural findings could be that this age group uses different brain areas or different underlying brain processes during the task. In line with this tentative suggestion, the whole-brain results showed there is activity in brain areas outside the regions of interest. In an earlier study by Fett et al. (2012), it was found that there was an age-related decrease of trust behavior accompanied with an age-related increase of TPJ activity when interacting with an untrustworthy partner (age 13-49). Also, in another study a positive association between age and TPJ activity during an untrustworthy interaction was found (age 16-27: Lemmers-Jansen et al., 2017). We speculate that young adolescents may show trust behavior but accompanying neural activity in areas of the mentalizing network is only found at a later age because younger adolescents may be using different brain areas to evaluate trustworthiness in the task.

When receiving the partner's feedback, increased activity in the reward and the cognitive control network, but not in the mentalizing network was observed. The reward network includes the bilateral caudate. In an earlier study, an age-related decrease of caudate activity was found when interacting with a trustworthy partner (Fett et al., 2012). It was suggested that caudate activity 
is reduced when outcomes are expected, and as people generally desire cooperation, the partner's trustworthy behavior may have been expected (Fett et al., 2012). In other studies it was also found that caudate activity is reduced when expectations and outcome match (Delgado et al., 2005; Schiffer \& Schubotz, 2011). In line with these findings, we suggest that activity in reward related areas may increase when reward and feedback processes are unexpected and unpredictable as might be with the untrustworthy behavior in the current study.

The cognitive control network, comprising the ACC and bilateral dlPFC in our study, is involved in conflict monitoring and cognitive control processes (Botvinick et al., 2004; Greene et al., 2004; MacDonald et al., 2000; Pochon et al., 2008; Yeung, 2014). The increased engagement of this network may indicate conflict between the desire of cooperation while the partner behaved untrustworthily. In an earlier study it was also found that, ACC activity increases with age during trust decisions when playing with an untrustworthy partner (Fett et al., 2012). Furthermore, agerelated increases in ACC activity and trend level age-related increases in dIPFC activity were found in another study during trust decisions during interactions with an untrustworthy partner (age 1319: Lemmers-Jansen et al., 2019). Although conflict monitoring and cognitive control processes continue to develop throughout adolescence (Crone \& Dahl, 2012; Crone \& Steinbeis, 2017; Kelly et al., 2009; Steinbeis et al., 2016), the results of the current study suggest that these processes are to some extent already present at the age of 12 .

\section{Relationship between trust behavior, neural mechanisms of trust and the social network position}

The second research aim was to examine the relationship between young adolescent's trust behavior, and the neural mechanisms of trust and real-life friendships. In line with our hypothesis, results indicated that more central social network positions are positively associated with overall 
trust behavior. In contrast to our hypothesis, we did not find an effect of eigencentrality on the ability to adapt trust behavior. Furthermore, the positive association between eigencentrality and baseline trust was borderline significant. Based on the power-dependence relation theory, we expected adolescents with a more peripheral position to sooner recognize the partner's untrustworthy behavior and in response to this, show a greater decrease of trust behavior and, consequently, show less overall trusting behavior. However, we did not find this greater decrease in trust behavior so it is difficult to conclude that the lower overall trust behavior is a consequence of a better ability to adapt to the partner's untrustworthy behavior. Also, the trend result showing that less central adolescents show lower baseline trust might suggest that the behavior at the beginning of the game influences the differences in overall trust behavior. In contrast, in our previous paper we did find that adolescents with more peripheral positions show a greater adaptive ability, as shown by a greater increase in trust behavior, compared to adolescents with more central positions (Sijtsma et al., 2020). This discrepancy might be due to differences in the experimental paradigms as we previously used information to inform the participants about the partner's trustworthiness before the game started. Importantly, the adolescents in our previous study were 16 to 18 years old while the adolescents in the current study were around 12 years of age, possibly suggesting that the association between adaptation of trust behavior and social network positions becomes apparent at an older age, when friendships are more established.

Contrary to our prediction, eigencentrality was not related to brain activity in the mentalizing network during the investment phase. This could be due to the overall insignificant findings during the investment phase. However, exploratory analyses also showed no associations between social network position and brain activity in the other two networks during the investment 
phase and no association between network position and brain activity in the three networks during the feedback phase.

\section{Limitations and future directions}

First, although our sample size was rather big for overall group analyses, it may have been small to detect subtle individual differences associated with social network position. Furthermore, like other studies, the partner in the trust game was a computer animation. This enables the use of a game without deception, but possibly makes the social interaction less realistic. Also, we examined a change of trust behavior over time, but we did not investigate brain activity changes over time. The current task was designed to detect changes in average brain activity over trials and is less suited to investigate changes in activity on trial-by-trial basis. Future studies may incorporate experimental designs that are better suited to investigate brain activity changes over the course of the task itself.

\section{Conclusion}

The current study indicates that young adolescents are able to decrease their trust behavior in response to untrustworthy behavior of others. No increased brain activity was found during trust decisions, however brain areas associated with cognitive control and reward processes were increasingly active when receiving feedback. Furthermore, the centrality of social network positions was positively related to overall trust behavior but not to brain activity. The current study adds to the literature by examining the neural mechanisms of trust in young adolescents and relating this to the daily-life friendships 
Friendships, trust and neural mechanisms

\section{Funding}

This study was supported by the European Research Council (ERC Consolidator grant, grant number 648082, to L.K.).

\section{Acknowledgements}

The authors thank Sanne Riemsma and Iris Vegting for their assistance with data acquisition, and all the adolescents, parents and schools for their participation. 
Friendships, trust and neural mechanisms

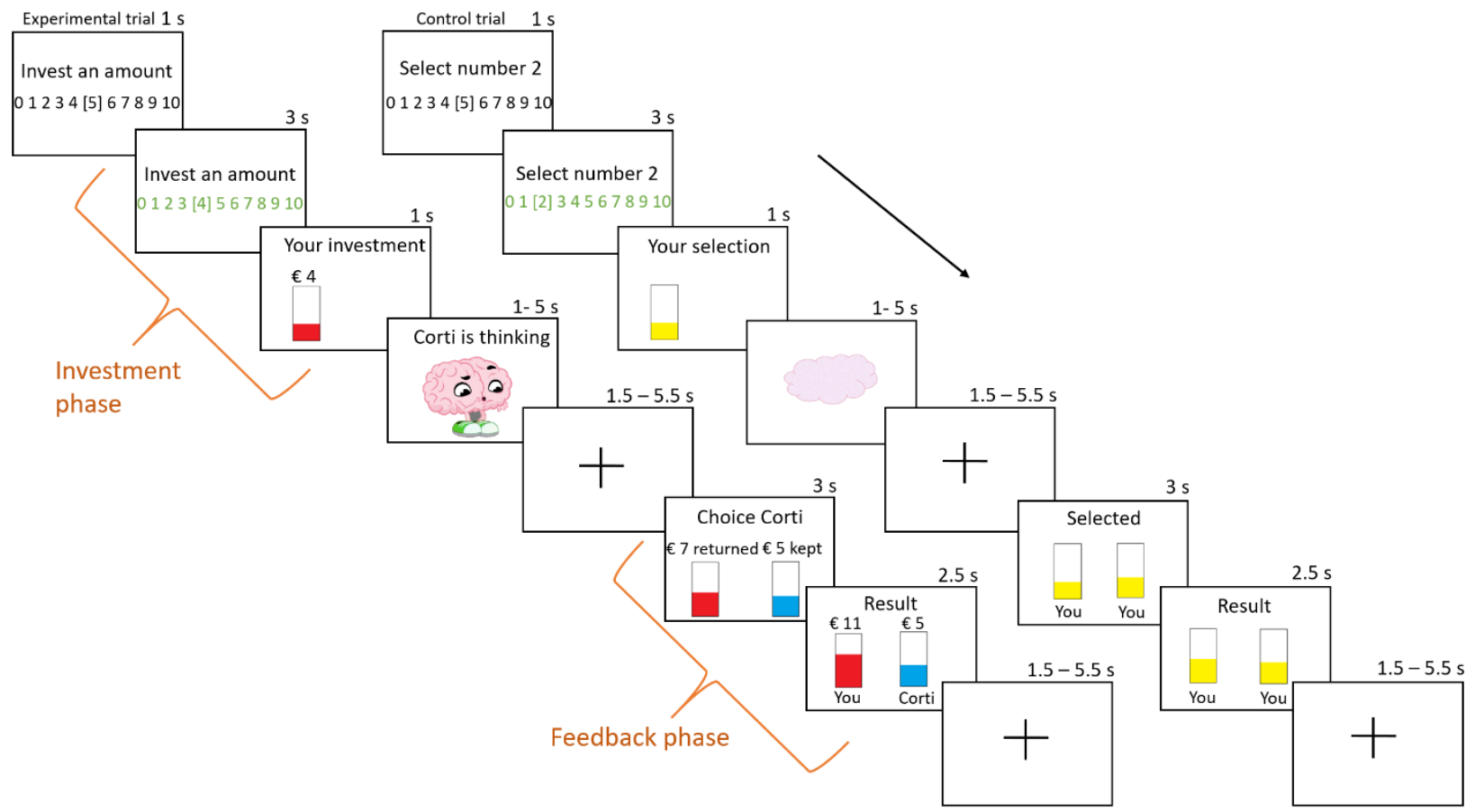

Figure 1: an experimental trial (left) and a control trial (right). The seconds above each screen indicate the durations. 
Friendships, trust and neural mechanisms

Table 1

Results model building procedure

\begin{tabular}{cllll}
\hline & Null model & Model 1 & Model 2 & Model 3 \\
\hline & AIC BIC & AIC BIC & AIC BIC & AIC BIC \\
\hline Model building procedure & 62296245 & 61876218 & 61816212 & 61706212 \\
\hline
\end{tabular}


Friendships, trust and neural mechanisms

Table 2

Results fit model 3

$\begin{array}{llll}\text { Variance } & \mathrm{B} & \mathrm{T} \text { value (p-value) } & 95 \% \mathrm{CI}\end{array}$

Lower Upper

Random effects

Intercept subjects

1.563

Slope time

0.003

Fixed effects

Intercept

$4.02 * *$

$22.258(<0.01) \quad 3.659$

4.381

Time linear

$-16.23 * *$

$-5.199(<0.01)$

$-22.47$

$-9.99$

Eigencentrality

$0.736^{* *}$

$4.074(<0.01)$

0.375

1.097

Cross-level interactions

Time linear*Eigencentrality

$-0.768$

$-0.246(0.81)$

$-7.011$

5.474

*two-tailed, $\mathrm{p}<0.05 . * *$ two-tailed, $\mathrm{p}<0.01$. 
A.

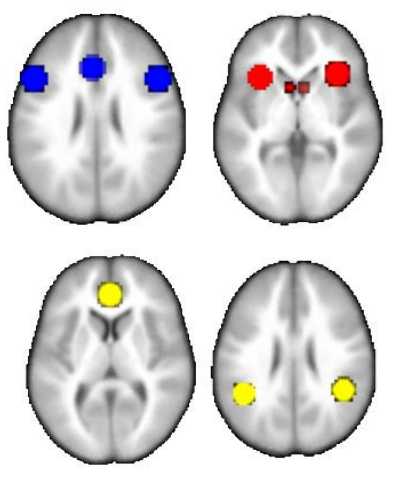

B.

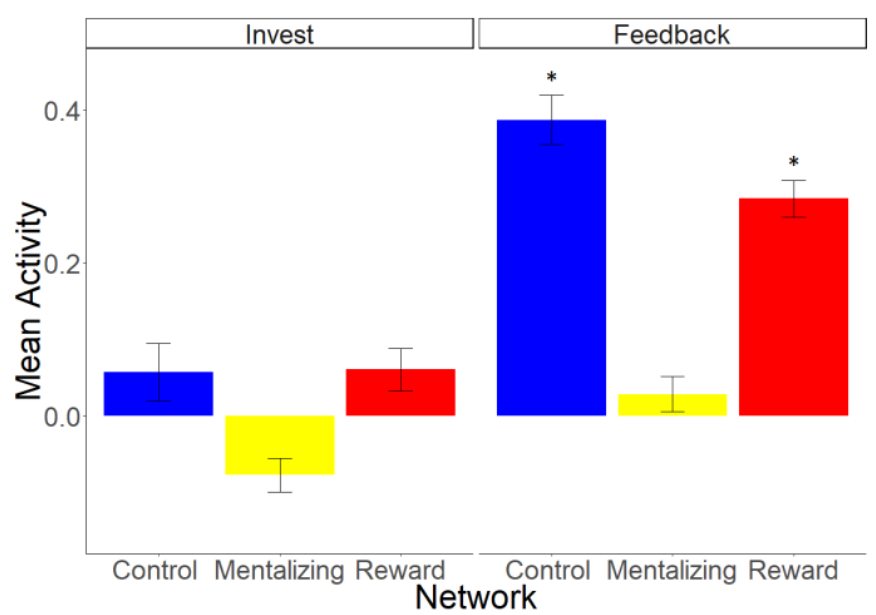

Figure 2: A: Networks of interest, blue=control network, yellow=mentalizing network, red=reward network. B: No significant activity during the investment phase, significant activity in the cognitive control network and the reward network during the feedback phase. 


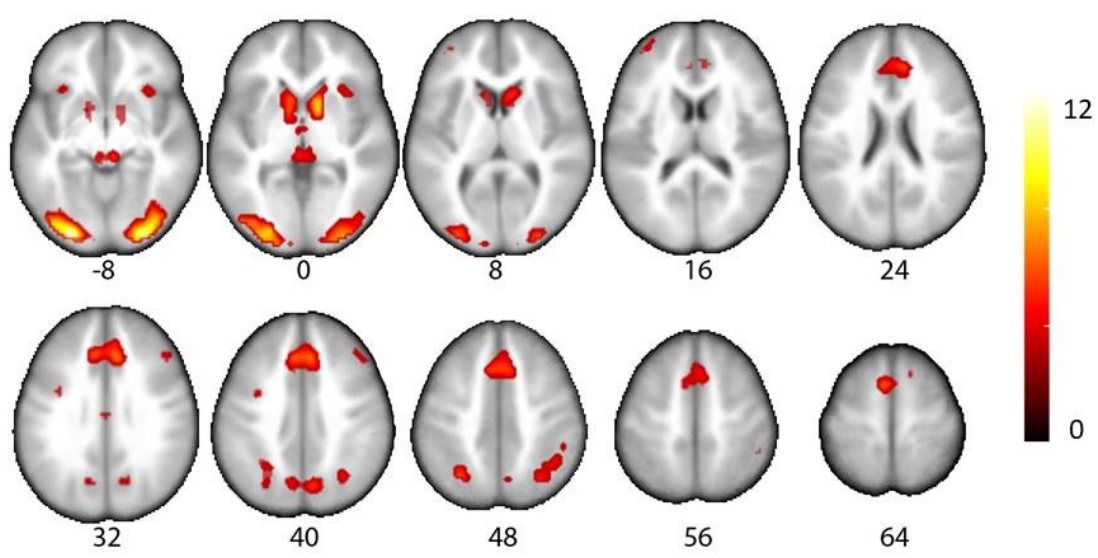

Figure 3: Results whole-brain analysis investment phase (cluster defining threshold of $\mathrm{p}<0.001$, cluster probability of $\mathrm{p}<0.05$, family wise error corrected). Activity changes are overlaid on an average anatomical brain obtain with Cerebromatic toolbox, matched for the age and gender of our subject sample. Color bars indicates t-values. 


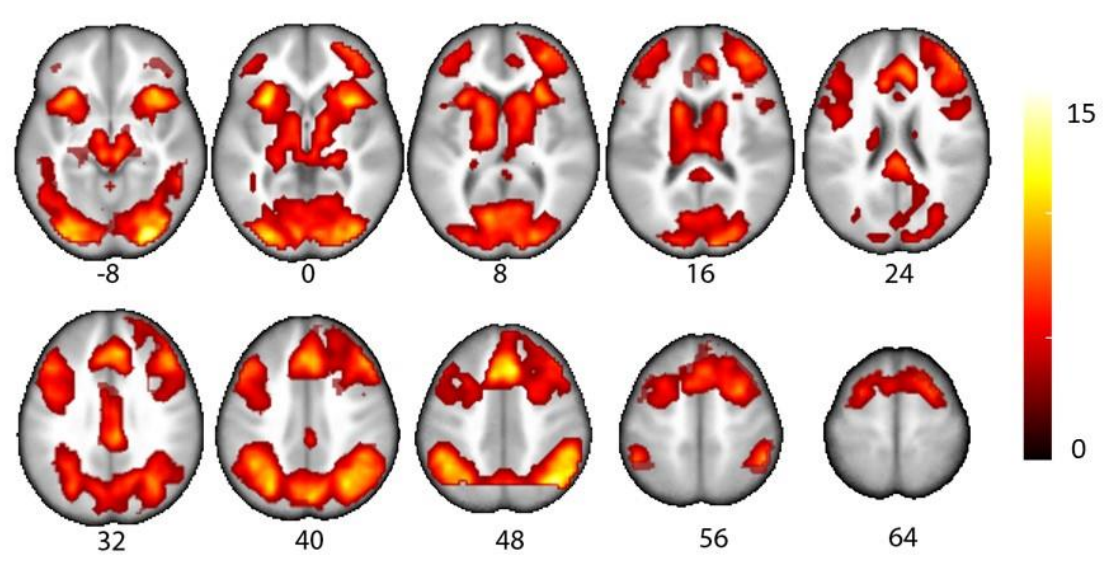

Figure 4: Results whole-brain analysis feedback phase (cluster defining threshold of $p<0.001$, cluster probability of $\mathrm{p}<0.05$, family wise error corrected). Activity changes are overlaid on an average anatomical brain obtain with Cerebromatic toolbox, matched for the age and gender of our subject sample. Color bars indicates t-values. 
A.

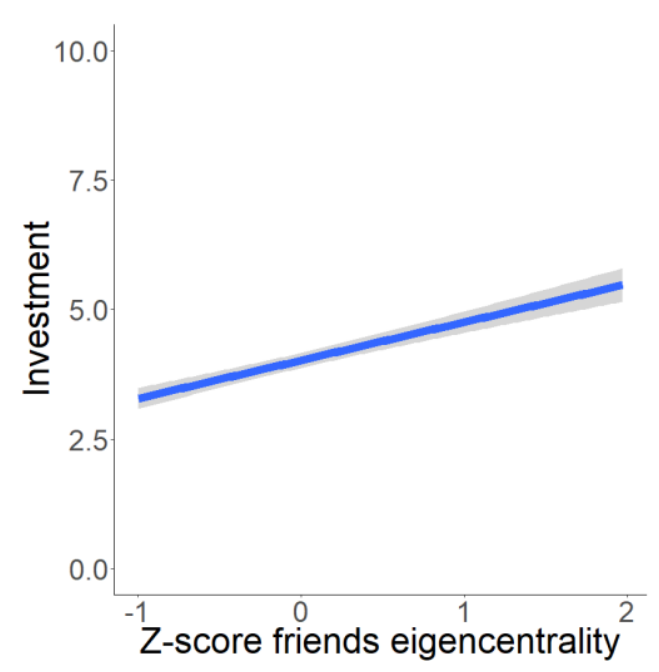

B.

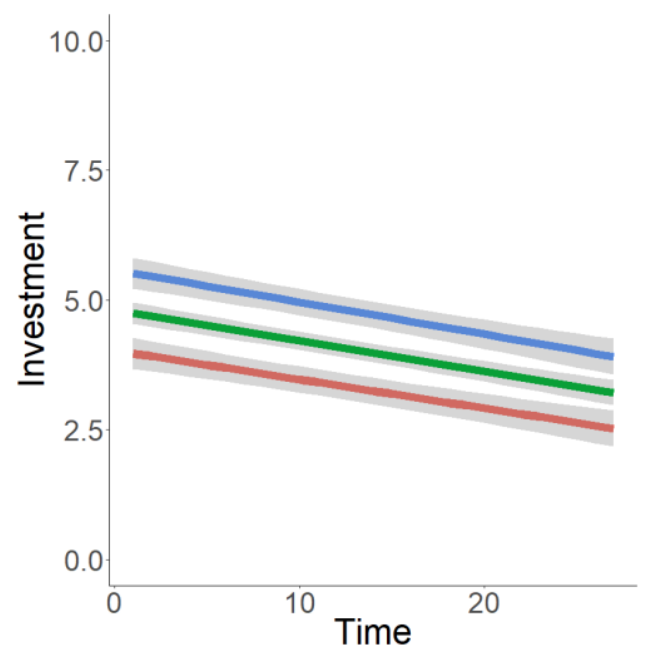

Figure 5: A: A positive effect of eigencentrality on the overall investments. B: No effect of eigencentrality on the change of investments over time. 
Friendships, trust and neural mechanisms

Legend Figure 5B

ZScore_Friends

= -1

1 
Friendships, trust and neural mechanisms

Table for supplementary materials

Number of participants and pupils per class

\begin{tabular}{|c|c|c|c|}
\hline Class & $\begin{array}{l}\text { Number of } \\
\text { participants per class }\end{array}$ & $\begin{array}{l}\text { Number of pupils per } \\
\text { class }\end{array}$ & $\begin{array}{l}\text { Percentage of } \\
\text { participants per class }\end{array}$ \\
\hline 1 & 24 & 28 & 0.86 \\
\hline 2 & 24 & 28 & 0.86 \\
\hline 3 & 25 & 29 & 0.86 \\
\hline 4 & 24 & 26 & 0.88 \\
\hline 5 & 25 & 27 & 0.93 \\
\hline 6 & 22 & 24 & 0.92 \\
\hline 7 & 23 & 26 & 0.88 \\
\hline 8 & 21 & 23 & 0.91 \\
\hline 9 & 25 & 30 & 0.83 \\
\hline 10 & 27 & 30 & 0.9 \\
\hline 11 & 27 & 30 & 0.9 \\
\hline 12 & 29 & 32 & 0.91 \\
\hline 13 & 22 & 28 & 0.79 \\
\hline 14 & 16 & 20 & 0.8 \\
\hline
\end{tabular}


Friendships, trust and neural mechanisms

Table for supplementary materials

Mean and standard deviation of investment per trial

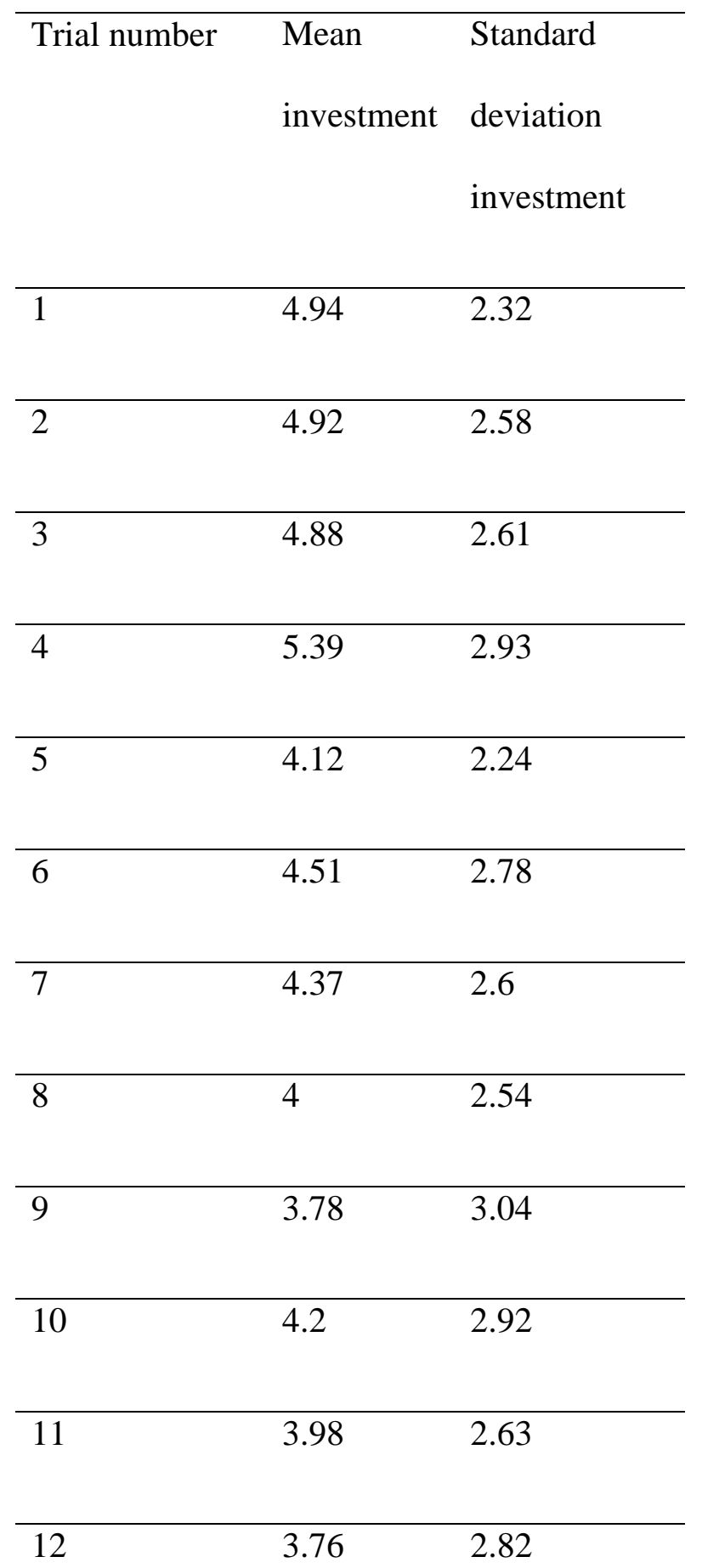


Friendships, trust and neural mechanisms

\begin{tabular}{|c|c|c|}
\hline 13 & 3.9 & 2.97 \\
\hline 14 & 4.35 & 3.17 \\
\hline 15 & 4.22 & 3.22 \\
\hline 16 & 3.71 & 3.12 \\
\hline 17 & 3.84 & 2.96 \\
\hline 18 & 3.39 & 2.86 \\
\hline 19 & 3.84 & 2.58 \\
\hline 20 & 3.67 & 2.44 \\
\hline 21 & 4.12 & 2.76 \\
\hline 22 & 3.61 & 2.64 \\
\hline 23 & 3.22 & 2.5 \\
\hline 24 & 3.45 & 2.69 \\
\hline 25 & 3.61 & 2.83 \\
\hline 26 & 3.31 & 3.12 \\
\hline 27 & 3.47 & 2.85 \\
\hline
\end{tabular}




\section{References}

Bates, D., Maechler, M., Bolker, B., \& Walker, S. (2015). Fitting linear mixed-effects model using Ime4. Journal of Statistical Software, 67, 1-48.

Berg, J., Dickhaut, J., \& McCabe, K. (1995). Trust, reciprocity, and social history. Games and Economic Behavior, 10(1), 122-142.

Bickart, K. C., Hollenbeck, M. C., Barrett, L. F., \& Dickerson, B. C. (2012). Intrinsic amygdala-cortical functional connectivity predicts social network size in humans. Journal of Neuroscience, 32(42), 14729-14741.

Borgatti, S. P., Everett, M. G., \& Freeman, L. C. (2002). Ucinet for windows: Software for social network analysis. Harvard, MA: Analytic Technologies.

Botvinick, M. M., Cohen, J. D., \& Carter, C. S. (2004). Conflict monitoring and anterior cingulate cortex: an update. Trends in Cognitive Sciences, 8(12), 539-546.

Brown, B. B., \& Larson, J. (2009). Peer Relationships in Adolescence. In Handbook of adolescent psychology (pp. 74-103). John Wiley \& Sons.

Burt, R. S., Kilduff, M., \& Tasselli, S. (2013). Social network analysis: Foundations and frontiers on advantage. Annual Review of Psychology, 64, 527-547.

Buskens, V. (1998). The social structure of trust. Social Networks, 20(3), 265-289.

Cillessen, A. H., \& Marks, P. E. (2011). Conceptualizing and measuring popularity. In Popularity in the peer system (pp. 25-56). Guilford Press.

Crone, E. A., \& Dahl, R. E. (2012). Understanding adolescence as a period of social-affective engagement and goal flexibility. Nature reviews neuroscience, 13(9), 636-650.

Crone, E. A., \& Steinbeis, N. (2017). Neural perspectives on cognitive control development during childhood and adolescence. Trends in Cognitive Sciences, 21(3), 205-215.

Delgado, M. R., Frank, R. H., \& Phelps, E. A. (2005). Perceptions of moral character modulate the neural systems of reward during the trust game. Nature Neuroscience, 8(11), 1611. 
Friendships, trust and neural mechanisms

Dunbar, R. I. (1998). The social brain hypothesis. Evolutionary Anthropology: Issues, News, and Reviews: Issues, News, and Reviews, 6(5), 178-190.

Emerson, R. M. (1962). Power-dependence relations. American Sociological Review, 31-41.

Falk, E. B., \& Bassett, D. S. (2017). Brain and social networks: fundamental building blocks of human experience. Trends in Cognitive Sciences, 21(9), 674-690.

Fett, A.-K. J., Gromann, P. M., Giampietro, V., Shergill, S. S., \& Krabbendam, L. (2012). Default distrust? An fMRI investigation of the neural development of trust and cooperation. Social Cognitive and Affective Neuroscience, 9(4), 395-402.

Fett, A.-K. J., Shergill, S. S., Gromann, P. M., Dumontheil, I., Blakemore, S.-J., Yakub, F., \& Krabbendam, L. (2014). Trust and social reciprocity in adolescence-a matter of perspective-taking. Journal of Adolescence, 37(2), 175-184.

Friston, K. J., Frith, C. D., Turner, R., \& Frackowiak, R. S. (1995). Characterizing evoked hemodynamics with fMRI. Neuroimage, 2(2), 157-165.

Galinsky, A. D., Magee, J. C., Inesi, M. E., \& Gruenfeld, D. H. (2006). Power and perspectives not taken. Psychological Science, 17(12), 1068-1074.

Galinsky, A. D., Rucker, D. D., \& Magee, J. C. (2016). Power and perspective-taking: A critical examination. Journal of Experimental Social Psychology, 67, 91-92.

Greene, J. D., Nystrom, L. E., Engell, A. D., Darley, J. M., \& Cohen, J. D. (2004). The neural bases of cognitive conflict and control in moral judgment. Neuron, 44(2), 389-400.

Kelly, A. C., Di Martino, A., Uddin, L. Q., Shehzad, Z., Gee, D. G., Reiss, P. T., Margulies, D. S., Castellanos, F. X., \& Milham, M. P. (2009). Development of anterior cingulate functional connectivity from late childhood to early adulthood. Cerebral cortex, 19(3), 640-657.

King-Casas, B., Tomlin, D., Anen, C., Camerer, C. F., Quartz, S. R., \& Montague, P. R. (2005). Getting to know you: reputation and trust in a two-person economic exchange. Science, 308(5718), 78-83.

Kuznetsova, A., Brockhoff, P. B., \& Christensen, R. H. B. (2017). ImerTest Package: Tests in Linear Mixed Effects Models. Journal of Statistical Software, 82, 1-26. 
Friendships, trust and neural mechanisms

Lee, N. C., Jolles, J., \& Krabbendam, L. (2016). Social information influences trust behaviour in adolescents. Journal of Adolescence, 46, 66-75.

Lemmers-Jansen, I. L., Fett, A.-K. J., Shergill, S. S., Van Kesteren, M. T., \& Krabbendam, L. (2019). Girlsboys an investigation of gender differences in the behavioral and neural mechanisms of trust and reciprocity in adolescence. Frontiers in Human Neuroscience, 13, 780-790.

Lemmers-Jansen, I. L., Krabbendam, L., Veltman, D. J., \& Fett, A.-K. J. (2017). Boys vs. girls: Gender differences in the neural development of trust and reciprocity depend on social context. Developmental Cognitive Neuroscience, 25, 235-245.

MacDonald, A. W., Cohen, J. D., Stenger, V. A., \& Carter, C. S. (2000). Dissociating the role of the dorsolateral prefrontal and anterior cingulate cortex in cognitive control. Science, 288(5472), 1835-1838.

Marks, P. E., Babcock, B., Cillessen, A. H., \& Crick, N. R. (2013). The effects of participation rate on the internal reliability of peer nomination measures. Social Development, 22(3), 609-622.

Pochon, J.-B., Riis, J., Sanfey, A. G., Nystrom, L. E., \& Cohen, J. D. (2008). Functional imaging of decision conflict. Journal of Neuroscience, 28(13), 3468-3473.

Powell, J., Lewis, P. A., Roberts, N., Garcia-Finana, M., \& Dunbar, R. I. (2012). Orbital prefrontal cortex volume predicts social network size: an imaging study of individual differences in humans. Proceedings of the Royal Society B: Biological Sciences, 279(1736), 2157-2162.

Robins, G. (2015). Doing social network research: Network-based research design for social scientists. Sage.

Schiffer, A.-M., \& Schubotz, R. I. (2011). Caudate nucleus signals for breaches of expectation in a movement observation paradigm. Frontiers in Human Neuroscience, 5, 38.

Schmälzle, R., O’Donnell, M. B., Garcia, J. O., Cascio, C. N., Bayer, J., Bassett, D. S., Vettel, J. M., \& Falk, E. B. (2017). Brain connectivity dynamics during social interaction reflect social network structure. Proceedings of the National Academy of Sciences, 114(20), 5153-5158.

Sijtsma, H., Lee, N. C., van Kesteren, M. T. R., van Buuren, M., Braams, B. R., van Atteveldt, N. M., \& Krabbendam, L. (2020). The adaptation of trust behavior and the association with friendships in adolescents. Pre-print on PsyArXiv. 
Friendships, trust and neural mechanisms

Steinbeis, N., Haushofer, J., Fehr, E., \& Singer, T. (2016). Development of behavioral control and associated vmPFC-DLPFC connectivity explains children's increased resistance to temptation in intertemporal choice. Cerebral cortex, 26(1), 32-42.

Steinberg, L., \& Morris, A. S. (2001). Adolescent development. Annual Review of Psychology, 52(1), 83110.

Sutter, M., \& Kocher, M. G. (2007). Trust and trustworthiness across different age groups. Games and Economic Behavior, 59(2), 364-382.

van den Bos, W., Crone, E. A., Meuwese, R., \& Güroğlu, B. (2018). Social network cohesion in school classes promotes prosocial behavior. PloS One, 13(4), 1-16.

Van den Bos, W., van Dijk, E., \& Crone, E. A. (2011). Learning whom to trust in repeated social interactions: A developmental perspective. Group Processes \& Intergroup Relations, 15(2), 243256.

Van Den Bos, W., van Dijk, E., Westenberg, M., Rombouts, S. A., \& Crone, E. A. (2009). What motivates repayment? Neural correlates of reciprocity in the Trust Game. Social Cognitive and Affective Neuroscience, 4(3), 294-304.

van den Bos, W., van Dijk, E., Westenberg, M., Rombouts, S. A., \& Crone, E. A. (2011). Changing brains, changing perspectives: The neurocognitive development of reciprocity. Psychological Science, 22(1), 60-70.

van den Bos, W., Westenberg, M., van Dijk, E., \& Crone, E. A. (2010). Development of trust and reciprocity in adolescence. Cognitive Development, 25(1), 90-102.

Wilke, M., Altaye, M., Holland, S. K., \& Consortium, C. A. (2017). CerebroMatic: a versatile toolbox for spline-based MRI template creation. Frontiers in computational neuroscience, 11, 5.

Yeung, N. (2014). Conflict monitoring and cognitive control. In Oxford library of psychology. The oxford handbook of cognitive neuroscience. (pp. 275-299). Oxford University Press. 